\title{
SENSOR BASED ALGORITHMS FOR DEAD RECKONING: A COMPARISON OF TWO APPROACHES FOR SMARTPHONES
}

\author{
Daniel Caspari ${ }^{1}$, Mircea Strutu ${ }^{2)}$, Lucas Riedhammer ${ }^{1)}$, Uwe Grossmann ${ }^{1)}$ \\ 1) Fachhochschule Dortmund, Emil-Figge-Strasse 44, www.mbms.fh-dortmund.de \\ \{daniel.caspari, uwe.grossmann\}@fh-dortmund.de, lucas-cedric.riedhammer001@stud.fh-dortmund.de \\ 2) Politehnica University of Bucharest, Splaiul Independentei 313, strutu_mircea@yahoo.com,www.upb.ro
}

\begin{abstract}
The implementation of a reliable indoor localization system can be the starting point for a variety of much desired applications. Either for efficient patient monitoring inside a hospital or as an automatic guide inside a museum, a working localization solution can be useful. Smartphone technology represents a powerful and user friendly tool in order to achieve adequate indoor positioning. This paper explores the potential of using smartphone sensor data (accelerometer and compass) in order to track the location of the person holding the device using dead reckoning algorithms. Two different approaches are under scrutiny in order to assess their performance in different real life inspired scenarios. Copyright (C) Research Institute for Intelligent Computer Systems, 2014. All rights reserved.
\end{abstract}

Keywords: Smartphone based indoor localization; dead reckoning; accelerometer sensors; fft; peak detection; smartphone; distance; indoor; positioning.

\section{INTRODUCTION}

Smartphone technology enables the possibility to develop a vast range of applications that can benefit from its ever increasing computing power, internet connectivity and interesting sensor capabilities. One of the problems that pose a great interest not only for private persons, but also for several public institutions is smartphone aided localization. This allows people to have an entirely new set of possibilities to interact with the surrounding environment by implementing location aware information systems.

Modern museums need to adapt to the needs and tastes of their guests. Therefore, one of the solutions they would like to have is a self-guidance system for smartphone devices. This would offer their visitors a great deal of freedom, giving them the possibility to move independently (without the aid of a human guide) and receive data about the exhibits they encounter, on their own familiar hand held device.

Assessing the gadget's location is no longer a problem for outdoor applications thanks to the advanced Global Positioning System (GPS). On the other hand, indoor localization does not benefit from the advantages of a universal approach.

Every indoor location has its own particularities regarding its geometry, construction materials, dimensions and wireless infrastructure. A vast diversity of approaches has been used in order to solve the localization problem in respect with each of the particular features listed above.

The only localization solution that holds regardless of the surrounding environment is dead reckoning (DR). In this case, the current position is determined by adding the distance travelled in a given time to the last known position, in the direction of movement. The travelled distance is a function of speed and time, while the direction of movement is determined using a compass [19].

This method was formerly used in seafaring, and has only recently been used for pedestrians, based on foot-mounted accelerometers $[10,17]$. In the latter case, it uses acceleration readings for step recognition.

The reason why this method is more general than the others is that it only relies on the integrated sensors of the mobile device. This consequently implies that there is no need for a special setting and its' only limitations are related to the sensor's accuracy and the necessary processing power to run the localization algorithm.

The purpose of this article is to determine if such a smartphone based localization system can be useful for indoor applications. In order to do so, two localization methods for dead reckoning have been considered. In this paper they are called Peak and FFT (Fast Fourier Transformation based method). Both methods are based on a given step size and a determined starting point. The performance analysis 
takes into account that the algorithms may perform differently from one scenario to another.

Therefore, after a short description of the two methods, a series of demonstrative tests will be depicted in order to determine if this is indeed a working solution, capable of fairly accurate indoor positioning. Next, a series of tests designed to determine the strengths and weaknesses of each algorithm will be described. These will include testing with different walking velocities, different holding positions or even different devices. A comparative statistical analysis will be available. Finally, the presented data will be evaluated and conclusions will be drawn.

\section{RELATED WORK}

Several implementation methods for localization have been discussed. Some popular approaches include signal fingerprinting [11], triangulation [3] and dead reckoning [2]. Each of the proposed solutions has its strengths and weaknesses.

The first approach to this problem has been carried out using Wi-Fi signal fingerprinting. The previous experience from [6] showed that "adapted algorithms can be used in large and dynamically changing environments and multi-level buildings". As we can find out from [13], fingerprinting can be time consuming, and easily becomes invalidated when physical conditions change, e.g. the number of people in the vicinity or new office equipment, thereby requiring new measurements to keep the calibration database up to date.

The approach of dead reckoning is not completely new, but had been used before for gait pattern recognition [14]. A good overview was given by Harle [7]. The survey discusses the implementation of different Pedestrian Dead Reckoning (PDR) systems, their advantages and disadvantages. In addition, it gives a comprehensive overview of the existing step detection methods and describes the standardized Internal Navigation System (INS) solution for PDR.

Mikov et al. achieved good accuracy in estimating the travelled distance relying only on the accelerometer data [15]. However, their results are based on data gathered using a special device, employing a non-standard sensor.

Strutu et al. presented a method which relies only on accelerometer and compass data, sensors which are generally available for virtually any smartphone [21]. Due to a step detection algorithm (based on peak determination), it was possible to assess the position of the person holding the device. During the determination of this technique's performance, it was assumed that the starting point of each path is known (by reading an NFC tag) and that the step length is dependent only to the individual's height. Also, the tests were only intended to present that steps can be determined if the smartphone is used in a hand held position.

Because these hypotheses were only covering a small part of the possible real life scenarios, the efforts have been concentrated on determining possible ways in which the algorithm can be used for more complex situations. In this regard, a new distance estimation method based on Fast Fourier Transformation was tested [4].

Thus, in this article, two methods of DR will be compared. The first one will be called Peak method and the other Fast Fourier (FFT) method. They both rely on the acceleration data provided by the acceleration sensor of an Android smartphone and use the provided compass data to estimate the direction of movement. However, as the method used for determining the walking direction is the same, this article focuses on the distance estimation.

\section{METHODS}

Dead reckoning (DR) "is the process of estimating the current position of a user based upon a previously known position, and advancing that position based upon measured or estimated speeds over elapsed time and course" [19]. For smartphones the method works using compass and acceleration sensors. The direction of movement of a smartphone holder can be obtained by its compass sensor, while the movement itself can be detected by the acceleration sensor. Analyzing the time signal of acceleration data yields steps frequencies. Combining these with the step length results in the velocity of the movement. Hence, travelled distance can be determined knowing the velocity and elapsed time.

The time signal of the acceleration sensor is recorded in three orthogonal directions (Fig. 1), left / right, forward / backward, up / down.

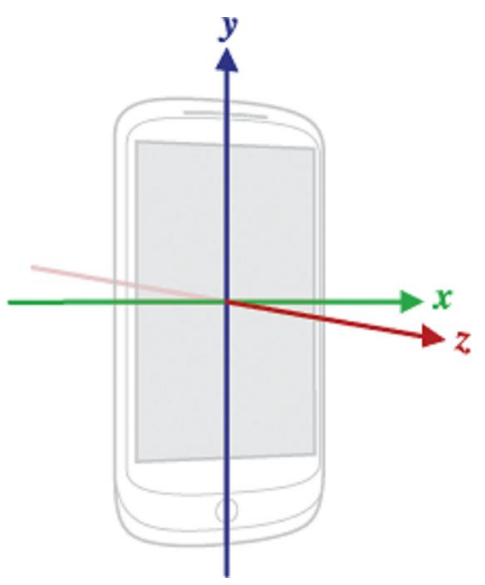

Fig. 1 - Axes of a smartphone [16]. 


\subsection{PEAK METHOD}

The Peak method uses the length of the acceleration vector $\left(m_{i}\right)$ as a measurement of the accelerometer data. The idea is that a step can be identified as a high peak in the time signal of the acceleration vectors. Assuming that there has to be a minimal time interval between two consecutive steps and that the measured acceleration in case of a step is significant larger than other possible accelerations (e.g. hand movement); a step is only counted when these requirements are met.

In real life scenarios there are different holding positions for a smartphone, meaning concentrating on only one axis is not useful. Hence, the time signal from all 3 acceleration axes is transferred into one corresponding value time signal using (1).

$$
m_{i}=\sqrt{x_{i}^{2}+y_{i}^{2}+z_{i}^{2}} ; \mathrm{i}=1, \ldots, \mathrm{k}
$$

Therefore, in order to have an estimation of the entire acceleration, the signal's magnitude is used (Fig. 2).

In order to get the correct number of steps using only accelerometer data, a series of checks are imposed that result in identifying the highest points (peaks) which consequently can be considered to be step marks. Using this method, step counting is possible. For each step walking direction should also be assessed (compass reading) in order to determine the user's path.

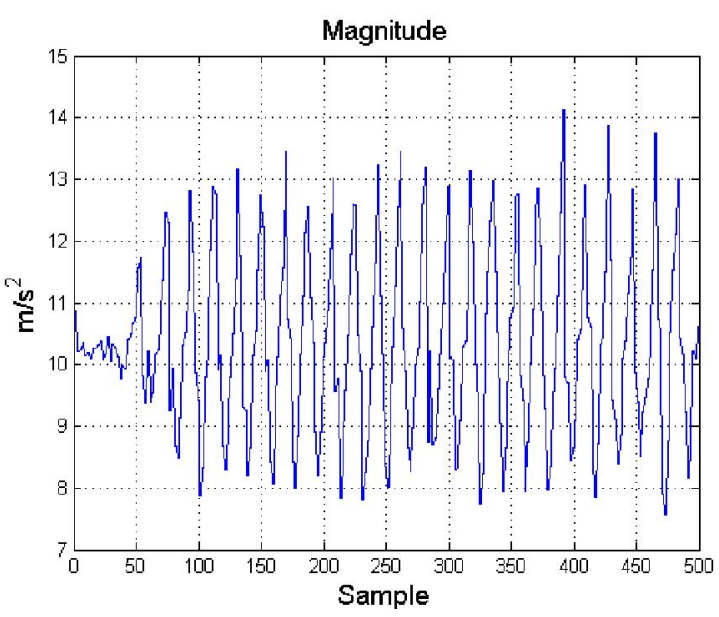

Fig. 2 - Length of acceleration vector vs. time.

Step Counting Algorithm

1. Start application - extract $\mathrm{x}, \mathrm{y}, \mathrm{z}$ (axis accelerometer data) and compass heading;

2. Calculate each sample's magnitude by using (1);

3. Determination of the first peak in the value time signal.
4. Comparing the value of the peak to a given threshold.

5. Comparing the value of the peak to a moving average threshold. The threshold consists of an average of a specific number of previous samples.

6. Comparing the time between this peak and the next to a given threshold.

7. The peak is considered to be a valid step.

8. The next peak in the value time signal is looked at and the algorithm starts at 4 .

If a sample validates all the conditions above, the number of steps is increased and an average heading is assigned to the newly found step. (The assigned heading is considered to be the average value of the heading registered by the last few samples before the peak and the peak itself).

The covered distance is calculated by using (2).

$$
d_{\text {peak }}=\operatorname{step}_{\text {count }} * \text { step }_{\text {size }},
$$

where $d_{\text {peak }}(\mathrm{cm})$ is the determined travelled distance, step $_{\text {count }}$ the number of identified steps and step $_{\text {size }}(\mathrm{cm})$ the assumed step size over the time interval.

\subsection{FFT METHOD}

Another approach for analyzing recorded acceleration values is based on transforming the time signal of acceleration data (1) firstly into a homogeny time acceleration signal and then into frequency domain using Fast Fourier Transformation. A moving time window of 2.56 or $3.2 \mathrm{sec}$ width is used, which slides over the time signal (see Fig. 3, upper part). The resolution is sample frequency through sample count per time window. The two red vertical bars indicate the current time window. The signal within this window will be transformed to frequency domain.

The idea is to determine a step frequency representative for one window as the dominant frequency (unique maximum) within the frequency range of $0-4 \mathrm{~Hz}$ of the according normalized frequency power spectrum (NFPS). The frequency power spectrum is normalized according to its maximum value within the $0-4 \mathrm{~Hz}$ interval (see Fig. 3, lower part). As normal step frequencies are below $3 \mathrm{~Hz}$ a range of $0-4 \mathrm{~Hz}$ (app. $10 \mathrm{~km} / \mathrm{h}$ ) should be sufficient. Furthermore, frequencies not belonging to the step frequency spectrum are filtered out.

If there is a NFPS value of 1 within the frequency range $0-4 \mathrm{~Hz}$ and no other frequencies exist with NFPS values above a given threshold value (see Fig. 3, lower part, red line), the frequency with 
NFPS value 1 is interpreted as the dominant frequency, and thus interpreted as the step frequency of the according time window. In any other case the frequency for this time window will be discarded. The percentage of discarded frequencies is about $10 \%$ for all tests.

This step frequency is then correlated to the starting point of the corresponding time window. If there is no unique maximum above the threshold value within the range of $0-4 \mathrm{~Hz}$, no step frequency can be determined. Figure 3 (middle part) shows the calculated step frequencies (CSF) over the already processed time interval.

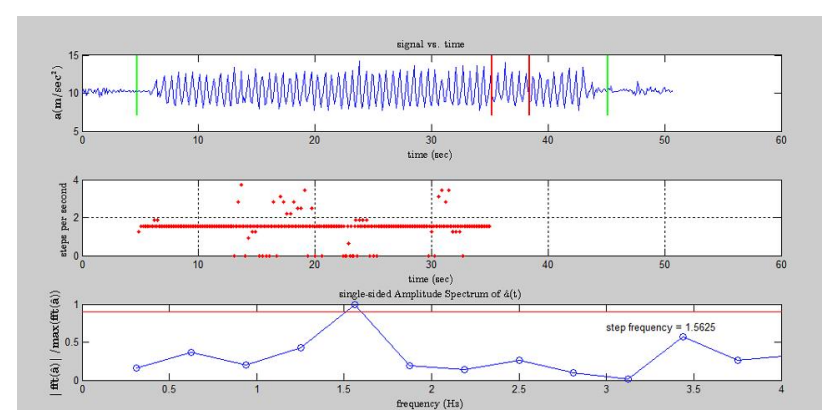

Fig. 3 - Acceleration-Time Signal (top), Calculated step frequency vs. time (middle), Normalized power frequency spectrum (bottom).

Dividing a received step frequency by the sampling rate of the time signal and then multiplying with the step size yields the covered distance between two sample time points. Formula (3) shows the accumulated covered distance over all time points $i$ of the time signal.

$$
d_{f f t}=\sum_{i} \frac{\text { step }_{f r e q}(i)}{f_{S}} * \text { step }_{\text {size }},
$$

where $d_{f f t}(\mathrm{~cm})$ represents the covered distance, $\operatorname{step}_{\text {freq }}(i)(\mathrm{Hz})$ the step frequency at time point i, step $_{\text {size }}(\mathrm{cm})$ the assumed average step size and $f_{s}(\mathrm{~Hz})$ the time signal's sampling rate.

\section{TEST ENVIRONMENT}

Tests have been carried out using two smartphones of different types (HTC One X; Samsung Galaxy Nexus) both with Android 4.3.2. In case of the accelerometer the HTC One X has the newer BMP250 while Samsung Galaxy Nexus has BMP220. The basic functionality of both sensors is the same, but the BMP250 is improved in terms of accuracy and output noise. For the exact differences please see the original data sheets provided by Bosch [4].
The developed test application records real time data from the smartphone acceleration sensor, together with a time stamp and offers the possibility to mark the time signal in order to emphasize different events during testing. The same data is used for both above mentioned methods and was processed with MatlabC.

The Android System provided the acceleration data but used a different library to access the acceleration sensor on each device. On HTC One X, the Android System uses "The Panasonic Android Open Source Project" library while, on Samsung Galaxy Nexus, the "Invensense Motion Processing Library" is used. As the test application uses the sensor data provided by the Android system, it must be assumed that the provided data is preprocessed by those libraries. Due to limitations of the Android System, the accelerometer sensor and compass cannot be used in order to achieve homogeneous sampling rates. The average sampling rate has been $10 \mathrm{~Hz}$.

Both devices are capable of Near Field Communication (NFC). NFC is working in close distance about 1-4 $\mathrm{cm}$. By reading a NFC tag with known position the position of the reading device is identified.

Since the building used for the initial tests was no longer available, later tests had been conducted in another indoor location (inside one of our university's buildings).

All tests were carried out by one person in order to minimize differences through different step characteristics.

\section{TESTS}

\subsection{INITIAL TESTS}

The initial tests had been conducted inside of a faculty building of the University of Applied Sciences and Arts Dortmund using a pathway of roughly 44 meters (Fig. 4). These were used as an experimental background for a previous paper [21], as proof of concept and using only the Peak method. For these tests a HTC One X smartphone has been used, which was held horizontally within the palm in front of the test person.

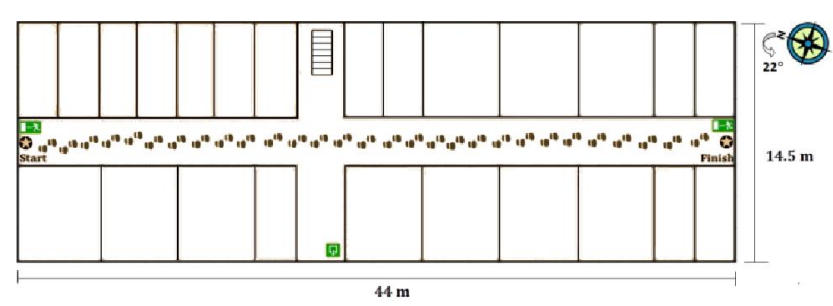

Fig. 4 - Test scenario with 60 steps in straight line (around 44 meters). 
The first experiments' goal has been to identify the values needed for the calibration parameters. In order to do so, 8 experiments were performed consisting of 60 steps in straight line having on average around 44.1 meters. The distance was calculated using (2) with an assumed step size of $0.73 \mathrm{~m}$. The average number of steps achieved is 59.75 with a calculated distance of $44.1 \mathrm{~m}$. The average error summing the error by compass offset and missed steps has been 3.1 meters (around 7\%). Also it was noticed that the device has a compass deviation of $3^{\circ}$ which can just be recalibrated. With this recalibration there was an error of 0.52 meters. Therefore, it can be concluded that with proper calibration, compass errors can be considerably reduced.

Next, a test scenario based on the same calibration parameters is shown. The test consists of 60 steps taken in straight direction with 2 missing steps. It is presented in Fig. 5. Using a peak algorithm implemented in Matlabఠ, it can be seen exactly which steps have been missed and the overall error (Fig. 5).

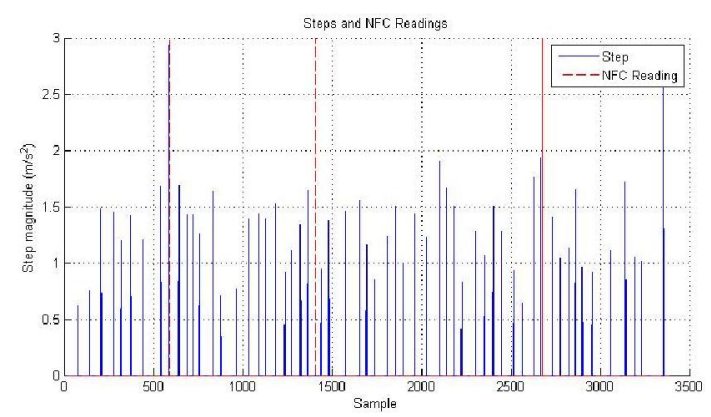

Fig. 5 - A graphical representation of steps in regard to their position inside the sample data vector and NFC tag readings.

NFC has been used in order to compare the results between tests with or without recalibration. Different numbers of (location recalibrating) NFC tag readings and tests with no NFC tag readings have been conducted.

The first case taken into account does not consider any NFC readings (Fig. 6). The number of steps is 58 with a calculated distance of $42.3 \mathrm{~m}$. The total difference between endpoint and calculated endpoint (DEP) is $3.07 \mathrm{~m}$.

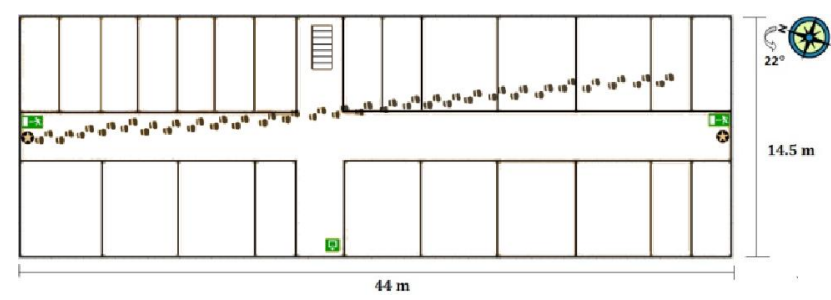

Fig. 6 - Tracking position without NFC tags.
In order to determine the benefit of NFC recalibration, as a second case one NFC tag has been used after 25 steps (Fig. 7). As the algorithm only covers for 24 steps and there is a heading error of around $3^{\circ}$, the NFC reading manages to correct this error. Overall, the DEP is now just $1.39 \mathrm{~m}$ (compared to $3.07 \mathrm{~m}$ without NFC).

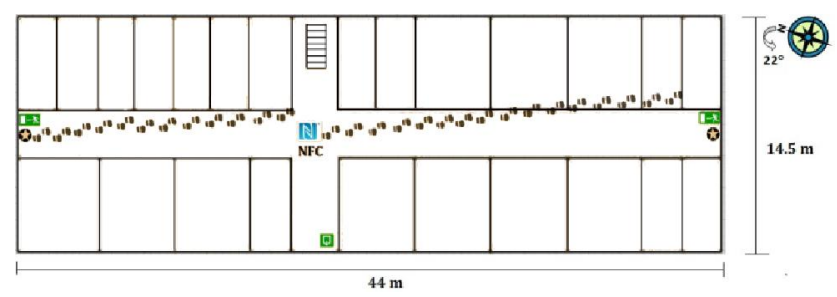

Fig. 7 - Tracking position using one NFC tag.

As third case, estimating the error by using 3 NFC tags positioned as depicted in Fig. 8 has been tested. The first NFC tag is positioned after 10 steps. As the algorithm only found 9 steps, it will help recover for the lost distance. The second NFC tag is positioned after 25 steps (out of which the algorithm can identify only 24) but, because the step loss occurred before the first NFC tag, the only remaining error is due to the heading offset. The third NFC tag also needs to account only for the heading error because there are no further lost steps. The DEP is $0.81 \mathrm{~m}$, mainly because of the lost step during the last interval. All the results are considered with no prior compass calibration.

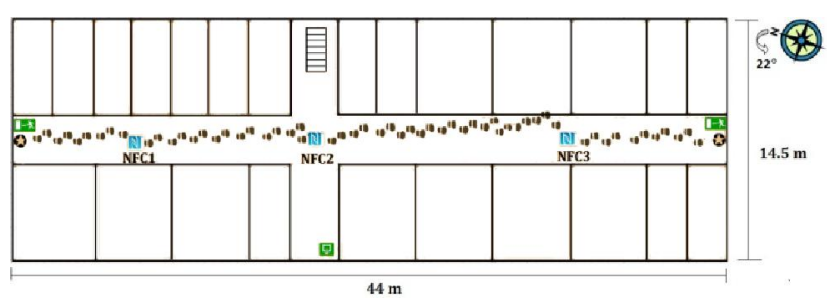

Fig. 8 - Tracking positions using 3 NFC tags.

Using NFC tags to reassess the location of the tester made a big difference in the overall error. This method is therefore useful and creates the opportunity to integrate it as part of the dead reckoning tracking system. The results of the experiments are highlighted in Table 1.

Table 1. Step Counting improved by NFC - 60 Steps.

\begin{tabular}{|c|c|c|c|c|c|}
\hline Case & $\begin{array}{c}\text { Detected } \\
\text { Steps }\end{array}$ & $\begin{array}{c}\text { Calculated } \\
\text { distance (m) }\end{array}$ & DEP (m) & $\begin{array}{c}\text { Error } \\
(\mathbf{\%})\end{array}$ & Remark \\
\hline $\mathbf{1}$ & 58 & 42.3 & 3.07 & 7 & No NFC Tag \\
\hline $\mathbf{2}$ & 58 & 42.3 & 1.39 & 3 & 1 NFC Tag \\
\hline $\mathbf{3}$ & 58 & 42.3 & 0.81 & 1 & 3 NFC Tags \\
\hline
\end{tabular}


The objective was to implement the localization system inside a large room where people are free to move in any direction. Therefore, it should provide accurate information even if the visitors would have a trajectory resembling the one depicted in Fig. 9.

The test shows how a more complicated trajectory can be tracked using the algorithm and gives a rough indication about the validity of the received data. The red line represents the real path and the green dots are the steps taken, therefore reconstructing the path.

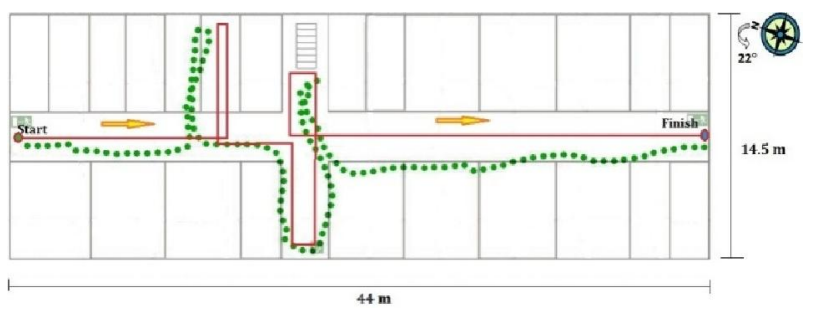

Fig. 9 - Simulated position using magnitude and compass readings in a scenario resembling a real life visitor activity.

Therefore, the initial tests conclusion has been that without using NFC tag readings, the determined location is not accurate enough in order to move through long distances or time. However, localization is possible for several meters, giving the visitor the possibility to freely enjoy the exhibits.

\subsection{COMPARING FFT ALGORITHM AND PEAK ALGORITHM}

The following test's purpose was comparing both algorithms regarding accuracy of determining step frequency and accumulated covered distance. As no changes at the parameter of the FFT method were made during the tests, the Peak method uses different thresholds for each device.

The test person walked along a straight line of 40 $\mathrm{m}$ length (Fig. 10, blue dotted line) with homogenous velocity and counted the performed steps. The mean step frequency (MSF) is calculated by dividing the counted steps by the walked time.

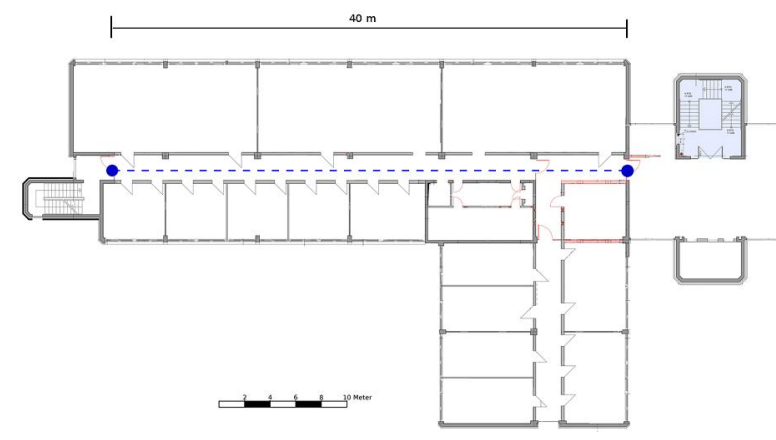

Fig. 10 - Testing area (office building), straight line of 40m length (dotted blue line).
The step frequency parameter is used as a base for comparison between the two algorithms, offering the possibility to directly evaluate their performances based on a common parameter. The Peak method provides a step frequency as the inverse of the time interval between two peaks, i.e. there is a step frequency for every peak time point. FFT provides step frequencies for every sample time point. According to sample rate and the width of the time window used, the FFT method yields a resolution of step frequencies of $0.3125 \mathrm{~Hz}$. In order to compare the results of both methods, the cumulative distribution of the ratio of calculated step frequency (CSF) to mean step frequency (MSF) is plotted.

There are several different test scenarios. All tests were carried out with both smartphones and at least twice.

The influences of three holding scenarios for the smartphones were examined. Firstly, the smartphone was held horizontally within the open hand in front of the test person. Secondly, the device was carried within the pocket of the test person's trousers. Thirdly, the test person was holding the smartphone in the hand, but swinging the arm as normal while walking and not watching the smartphone's display.

Moreover, we examined the influence of different velocities (slow/medium/fast). In case of slow velocity the test person needed 74 steps and $61 \mathrm{sec}$, i.e. velocity $=0.66 \mathrm{~m} / \mathrm{sec}$ and $\mathrm{MSF}=1.21 \mathrm{~Hz}$. In case of medium velocity it was 63 steps and $40 \mathrm{sec}$, i.e. velocity $=1.00 \mathrm{~m} / \mathrm{sec}$ and MSF $=1.58 \mathrm{~Hz}$, and in case of fast velocity $48 \mathrm{steps}$ and $28 \mathrm{sec}$, i.e. velocity $=1.42 \mathrm{~m} / \mathrm{sec}$ and $\mathrm{MSF}=1.71 \mathrm{~Hz}$.

The following figures present the ratio of CSF to MSF of both methods. The MSF is obtained by dividing the total number of steps counted during walking the test line by the total walking time.

\section{1) Comparing two devices}

This test was carried out with medium velocity and the smartphone held horizontally within the open hand in front of the test person.

Fig. 11 shows the results in case of using the Peak method with both devices. The first quartile shows a ratio of 0.65 in case of HTC One X and 0.7 in case of Samsung Galaxy Nexus. The second and third quartile shows a ratio of 0.85 respectively 0.95 for both devices. At $90 \%$ the ratio is 1 in case of HTC One X and 1.4 in case of Samsung Galaxy Nexus.

The Peak method is underestimating step frequency with both devices.

The results using the FFT method are presented in Fig. 12. The ratio of the first quartile shows 0.8 for both devices. The second quartile shows 0.9 in case of Samsung Galaxy Nexus and 0.95 in case of HTC One X. The third quartile shows 0.95 in case of 
Samsung Galaxy Nexus and 1 for HTC One X. At $90 \%$ the ratio shows 1 for Samsung Galaxy Nexus and 1.8 for HTC One X.

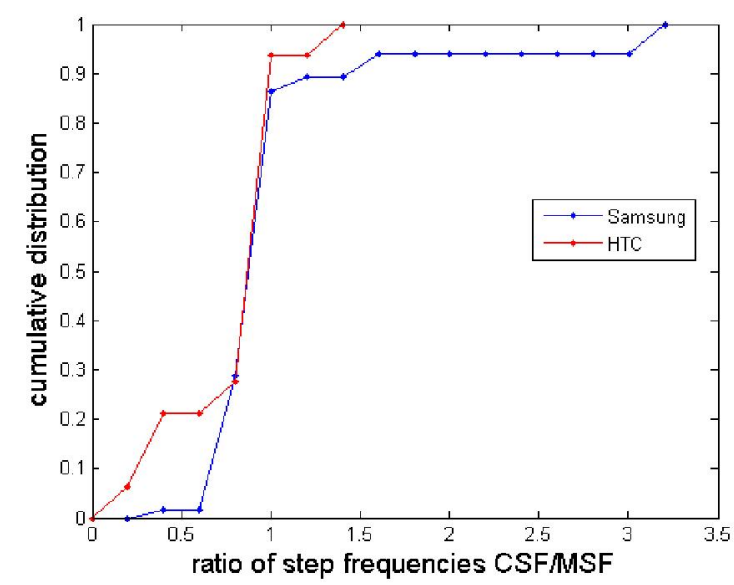

Fig. 11 - Peak method, medium velocity, HTC One X (MSF: 1.56Hz) and Samsung Galaxy Nexus (MSF: 1.49Hz).

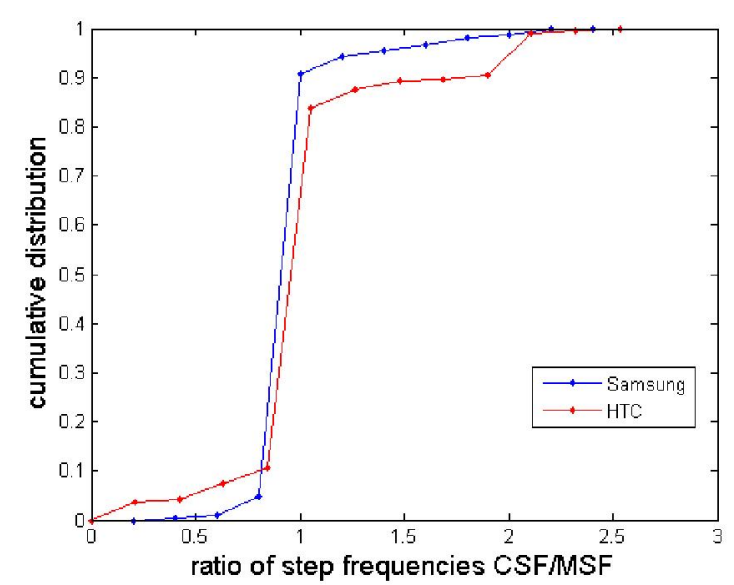

Fig. 12 - FFT method, medium velocity, HTC One X (MSF: $1.56 \mathrm{~Hz}$ ) and Samsung Galaxy Nexus (MSF: $1.49 \mathrm{~Hz})$.

The FFT method underestimates step frequencies, but not to the same amount as the Peak method.

As shown in Fig. 11 and Fig. 12 the ratio of CSF to MSF is more spread for the HTC One $\mathrm{X}$ than for the Samsung Galaxy Nexus.

Both devices are able to obtain a unique step frequency with both methods, albeit Samsung Galaxy Nexus performs better.

In the following presentation of results only the data obtained by Samsung Galaxy Nexus will be used. The data from HTC One X is more spread but essentially similar.

2) The influence of different velocities

The next figures show the results of tests using the Samsung Galaxy Nexus with three different velocities: slow (MSF: $1.21 \mathrm{~Hz}$ ), medium (MSF:
$1.58 \mathrm{~Hz}$ ) and fast (MSF: $1.71 \mathrm{~Hz}$ ) and the smartphone held horizontally within the open hand in front of the test person.

Fig. 13 shows the results of the Peak method.

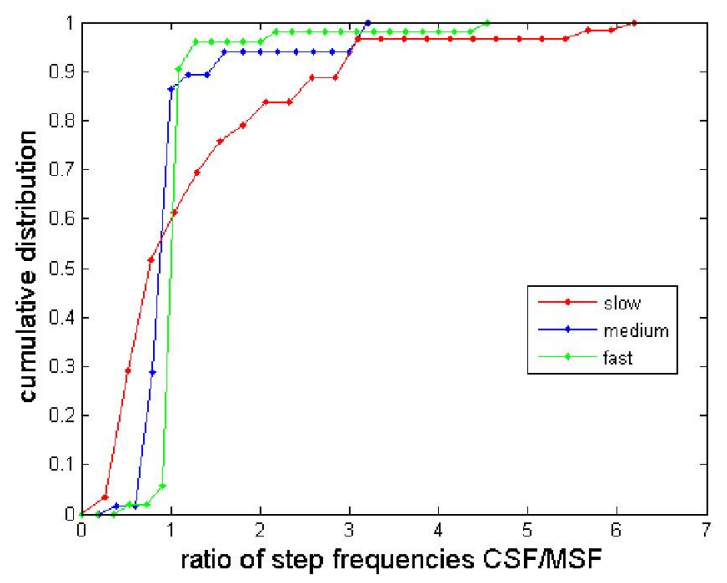

Fig. 13 - Peak method, different velocities, Samsung Galaxy Nexus.

The first quartile shows a ratio of $0.45,0.65$ and 0.9 in case of slow, medium and fast velocity. The second quartile shows a ratio of $0.7,0.8$ and 0.95 in case of slow, medium and fast velocity. The third quartile shows a ratio of 1.5, 0.9 and 1 in case of slow, medium and fast velocity. At $90 \%$ the ratio for slow, medium and fast velocity shows $2.8,1.4$ and 1.1 .

A nearly constant step frequency can be calculated while walking with medium and fast velocities, but the method is calculating varying step frequencies while walking slowly.

Fig. 14 presents the results using the FFT method. The first quartile shows a ratio of 0.9 in case of slow and medium and 0.95 in case of fast velocity. The second quartile shows a ratio of 0.95 in case of medium and 1 in case of slow and fast velocity. The third quartile shows a ratio of $1.8,1$ and 1.05 in case of slow, medium and fast velocity. At $90 \%$ the ratio for slow, medium and fast velocity shows 2.8, 1.05 and 1.1.

The FFT method is able to calculate distinct step frequencies while walking with medium and fast velocities, but with slow velocities results are more erratic.

As the Peak method is mostly underestimating and rarely overestimating, the FFT method is more overestimating. Furthermore, both methods have problems when used with slow velocities. Both methods perform almost equally in the case of medium and fast velocity.

3) Different holding positions of the smartphone

For these tests a medium velocity (MSF: 1.58 $\mathrm{Hz}$ ) was used. The smartphone was held either horizontally within the open hand in front of the test 
person (see Fig. 11 and 12) or it was held in the hand, but the arm was swinging as while walking (see Fig. 15) or the device was carried in the pocket (See Fig. 16).

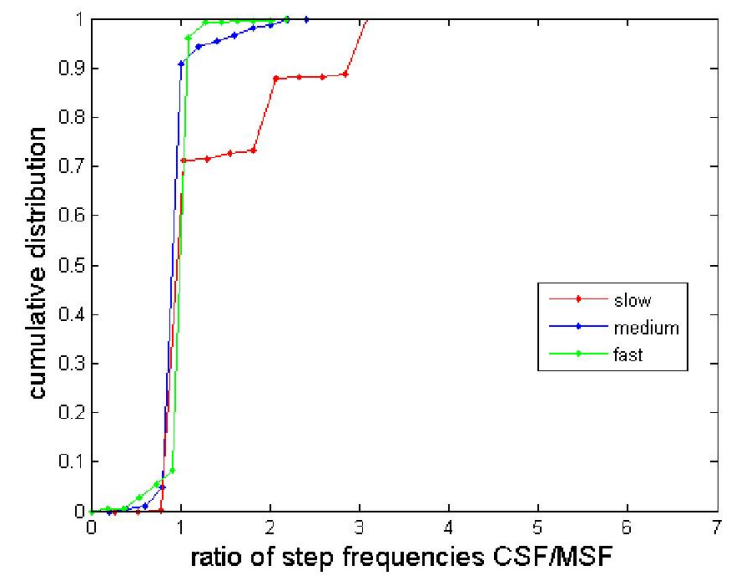

Fig. 14 - FFT method, different velocities, Samsung Galaxy Nexus.

Fig. 15 shows results in case of swinging the arm as during walking. This case represents walking without watching the display for the whole time.

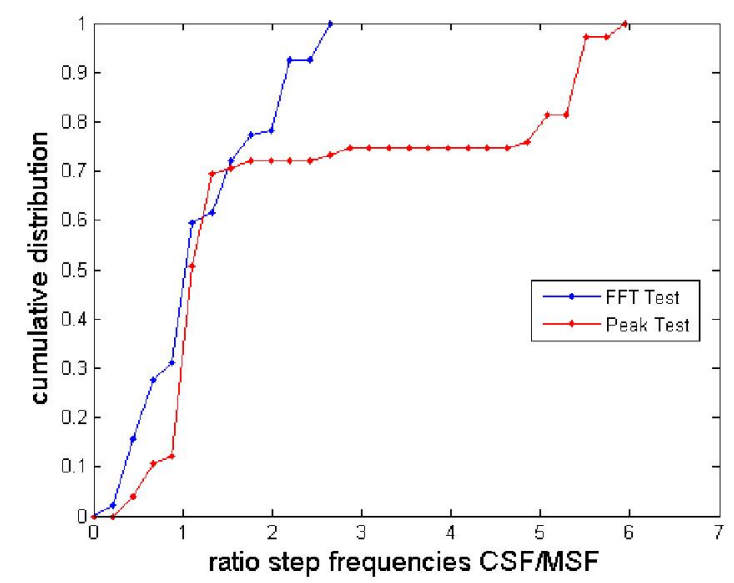

Fig. 15 - Peak and FFT method, arm swinging while walking, Samsung Galaxy Nexus.

In case of the Peak method the first quartile has a ratio of 1 while for the FFT method it shows 0.6. The second quartile shows a ratio of 1.05 in case of the Peak method and 1 for the FFT method. The third quartile shows a ratio of 5 in case of the Peak method and 1.6 in case of the FFT method. At 90\% the ratio of the Peak and the FFT methods is 5.4 and 2.2.

Fig. 16 shows the results for the case of carrying the device in the pocket of the test person's trousers.

At the first quartile the Peak method has a ratio of 1.2 while the FFT method has 0.4 . The second quartile shows a ratio of 1.5 for the Peak method and 1 for the FFT method. The third quartile shows a ratio of 4.6 in case of the Peak method and 1.05 in case of the FFT method. At $90 \%$ the ratio is 5.7 in case of the Peak method and 1.05 in case of the FFT method.

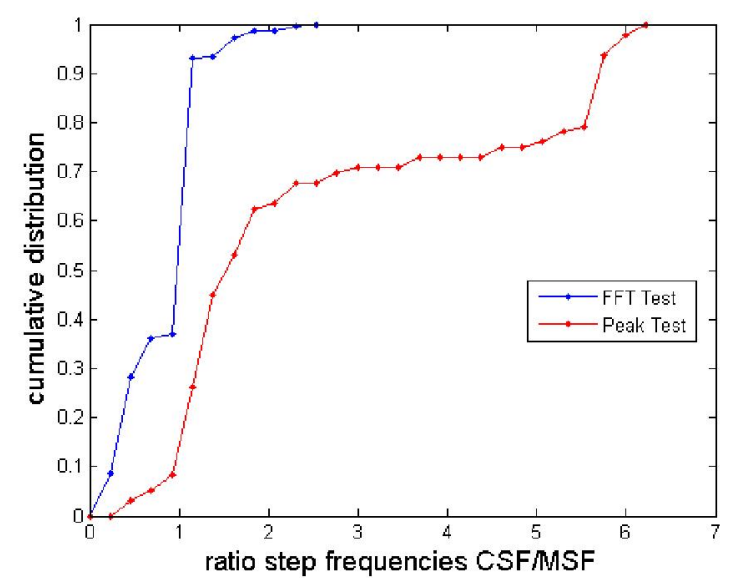

Fig. 16 - Peak and FFT method, device in the pocket, Samsung Galaxy Nexus.

For both tests, the FFT method performs better than the Peak method. While the FFT method underestimates in the results, the Peak method overestimates.

4) Comparing covered distance calculations of both methods

For comparing the resulting distances calculated by both methods one test was performed twice with medium velocity. The test consisted of walking a straight line of $40 \mathrm{~m}$ (see Fig. 10) with medium velocity. Performing this test several times shows differences of elapsed time of app. 1 sec. The measuring device was held in the horizontal position in front of the test person. In order not to interfere with problems related to the step size, the determined step length has been used, calculated from walked distance and counted steps by using formulas (2) and (3).

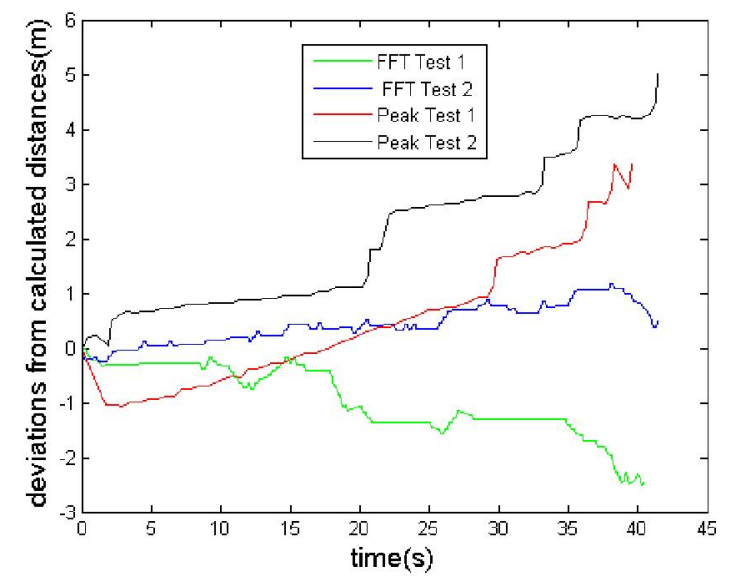

Fig. 17 - Time dependency of deviation of calculated distance from covered distance. 
Fig. 17 represents the deviation of calculated distance to covered distance over time. All tests show different progressions of deviation over time. Up to 18 seconds the absolute value of deviation is lower than $1 \mathrm{~m}$. After 20 seconds the absolute value of deviation tends to increase for both methods with Peak method overestimating the covered distance and FTT method partly underestimating covered distance.

\section{CONCLUSION AND FUTURE WORK}

The results lead to the conclusion that deadreckoning with both methods can be used, in principle, for smartphones as a positioning method between two secured positions. However, results diverge using either peak or FFT method or using different devices. The discrepancies of results in using different devices can be led back to the different accelerometer sensors of the two devices. Moreover, both devices uses different libraries to access the accelerometer data.

Furthermore results vary due to velocity. Both methods are more erratic with slow velocities. In addition the FFT method seems to perform better in calculating characteristic step frequencies. The FFT method seems to be more robust while walking with swinging the device or carrying the device in the pocket.

As shown by Fig. 17, the absolute value of deviations tends to increase over time, independent of the used method. Both methods estimate the covered distance with an accuracy of about $+/-1 \mathrm{~m}$ up to 16-20 seconds. After this time, the calculated positions should be considered as unreliable und need to be recalibrated by another method.

In comparison of both methods, distinctly more computing power is needed in case of the FFT method than in case of the Peak method. Though smartphones are capable of providing this computing power, the FFT method still has the disadvantage of reducing battery power more quickly.

Comparing to other papers the results seem to be reasonable. The results are partially worse, but the methods can be used to provide positioning. As Alzantot et al. [2] pointed out, using of the shelf smartphones for Dead Reckoning will lead to more errata. Alzantot et al. used standard smartphones among others, but get better results while walking with device swinging or in pocket. Like Mikov [15], if using slower walking velocities the results degrade. The results are comparable to those of Link [13] and Pratama [17].

The presented algorithms have the potential to work as a backup solution for movement tracking inside a large hall or a different indoor location where no other means of localization are possible. This offers the opportunity for a location aware information system to extend its applicability to areas which could not have been previously covered by other localization systems.

Our initial experiments suggest that the error can be kept inside tolerable margins if the position is e.g. periodically recalibrated using a NFC tag. However, the algorithm works even without NFC tag reading for a limited distance. Despite of the small range of $1-4 \mathrm{~cm}$, still some possible attack options on visitor devices are conceivable. An imaginable attack could be the manipulation of a NFC tag containing a target URL, which is used for content retrieval or positioning. Visitors could be directed to a website containing malicious software. This may be prevented by using Read-Only tags. Using NFC does not cause a significant threat for the smartphone.

In further experiments, the robustness of the method against the influence of more complex paths should be assessed. In these cases, changes of direction of movement will be included and the compass sensor needs to be analyzed and integrated into the method. Furthermore, possibilities of improving the resolution of the frequency spectrum should be investigated in order to improve differentiation of step frequency and thus speed accuracy.

\section{REFERENCES}

[1] D. Alvarez, R. C. Gonzalez, A. Lopez, J. C. Alvarez, Comparison of step length estimators from weareable accelerometer devices, Proceedings of the 28th IEEE Annual International Conference on Engineering in Medicine and Biology Society, EMBS'06, 2006, pp. 5964-5967

[2] M. Alzantot, M. Youssef, Uptime: ubiquitous pedestrian tracking using mobile phones, Proceedings of the IEEE Wireless Communications and Networking Conference (WCNC), 2012, pp. 3204-3209.

[3] A. Bahillo, S. Mazuelas, J. Prieto, P. Fernández, R.M. Lorenzo, and E.J. Abril, Hybrid RSS-RTT localization scheme for wireless networks, Proceedings of the IEEE International Conference on Indoor Positioning and Indoor Navigation (IPIN), 2010, pp. 1-7.

[4] Robert Bosch GmbH, Data Sheets, www.bosch-sensortech.com

[5] BMA220 http://ae-bst.resource.bosch.com/me dia/products/dokumente/bma220/BST-BMA 220-DS003-08.pdf 
[6] BMA250 http://ae-bst.resource.bosch.com/me dia/products/dokumente/bma250/bst-bma250ds002-05.pdf

[7] D. Caspari, J. Pickert, U. Grossmann, Positionsbestimmung mittels Dead Reckoning (Koppeln) und Schrittfrequenz-Erkennung, 10. Konferenz Wireless Communication and Information, 24./25.10.2013, Berlin, 2013.

[8] S. Gansemer, U. Grossmann, S. Hakobyan, RSSI-based Euclidean distance algorithm for indoor positioning adapted for the use in dynamically changing WLAN environments and multi-level buildings, Proceedings of the International Conference on Indoor Positioning and Indoor Navigation (IPIN), 2010, pp. 1-6.

[9] R. Harle, A survey of indoor inertial positioning systems for pedestrians, IEEE Communications Surveys \& Tutorials, (15) 3 (2013) pp. 1281-1293.

[10] Y. Hatano, Use of the pedometer for promoting daily walking exercise, Int. Council Health Phys. Educ. Recreat., (29) (1993), pp.4-8.

[11] J. Jahn, U. Batzer, J. Seitz, L. PatinoStudencka, J. Gutierrez Boronat, Comparison and evaluation of acceleration based step length estimators for handheld devices, Proceedings of the International Conference on Indoor Positioning and Indoor Navigation (IPIN), 2010, pp. 1-6.

[12] S. W. Jeen, W. L. Che, T. C. Yang, and J. H. Yu, "Walking pattern classification and walking distance estimation algorithms using gait phase information, IEEE Transactions on Biomedical Engineering, (59) 10 (2012), pp. 2884-2892.

[13] Y. Kim, Y. Chon, and H. Cha, Smartphonebased collaborative and autonomous radio fingerprinting, IEEE Transactions on Systems, Man, and Cybernetics, Part C: Applications and Reviews, (42) 1 (2012), pp. 112-122.

[14] H. Leppakoski, J. Collin, J. Takala, Pedestrian navigation based on inertial sensors, indoor map, and WLAN signals, Proceedings of the IEEE International Conference on Acoustics, Speech and Signal Processing (ICASSP), 2012, pp. 1569-1572.

[15] J.A.B. Link, P. Smith, N. Viol, and K. Wehrle, Footpath: Accurate map-based indoor navigation using smartphones, Proceedings of the IEEE International Conference on Indoor Positioning and Indoor Navigation (IPIN), 2011, pp. 1-8.

[16] J. Mantyjarv, M. Lindholm, E. Vildjiounaite, S.M. Makela, \& H.A. Ailisto, Identifying users of portable devices from gait pattern with accelerometers, Proceedings of the IEEE International Conference on Acoustics, Speech, and Signal Processing (ICASSP'05), March 2005, Vol. 2, pp. ii-973.

[17] A. Mikov, A. Moschevikin, A. Fedorov, and A. Sikora, A localization system using inertial measurement units from wireless commercial hand-held devices, Proceedings of the International Conference on Indoor Positioning and Indoor Navigation (IPIN'2013), October 2013, pp. 857-863.

[18] PmWiki, axis_device.png, from http://media 2mult.uos.de/pmwiki/fields/pgs10/index.php?n =Main.SensorenAndroid", 17.04.2013.

[19] A. Pratama, R. Widyawan, and R. Hidayat, Smartphone-based pedestrian dead reckoning as an indoor positioning system, Proceedings of the International Conference on System Engineering and Technology (ICSET), 2012, pp. $1-6$.

[20] T. Sayeed, A. Sama, A. Catala, J. Cabestany, Comparison and adaptation of step length and gait speed estimators from single belt worn accelerometer positioned on lateral side of the body, Proceedings of the IEEE $8^{\text {th }}$ International Symposium on Intelligent Signal Processing (WISP'13), 2013, pp. 14-20.

[21] A. Serra, D. Carboni, and V. Marotto, Indoor pedestrian navigation system using a modern smartphone pedestrian navigation system using a modern smartphone, Proceedings of the MobileHCI'2010, September 7-10, 2010, pp. 397-398.

[22] S. H. Shin, C. G. Park, J. W. Kim, H. S. Hong, J.M. Lee, Adaptive step length estimation algorithm using low-cost MEMS inertial sensors, Proceedings of the IEEE Sensors Applications Symposium SAS'07, 2007, pp. 1-5.

[23] M. Strutu, D. Caspari, J. Pickert, U. Grossmann, and D. Popescu, Pedestrian smartphone based localization for large indoor areas, Proceedings of the $7^{\text {th }}$ IEEE International Conference on Intelligent Data Acquisition and Advanced Computing Systems: Technology and Applications, Berlin, 12-14 September, 2013, pp. 450-454. 